\section{Both exogenous and endogenous target salience manipulations support resource depletion accounts of the attentional blink: A reply to Olivers, Spalek, Kawahara, and Di Lollo (2009)}

\author{
Paul E. Dux, Christopher L. Asplund, \\ AND RENÉ MAROIS \\ Vanderbilt University, Nashville, Tennessee
}

\begin{abstract}
Input control theories of the attentional blink $(A B)$ suggest that this deficit results from impaired attentional selection caused by the post-Target 1 (T1) distractor (Di Lollo, Kawahara, Ghorashi, \& Enns, 2005; Olivers, van der Stigchel, \& Hulleman, 2007). Accordingly, these theories predict that there should be no $A B$ when no distractors intervene between the targets. Contrary to these hypotheses, Dux, Asplund, and Marois (2008) observed an $A B$ (T3 deficit) when three targets, from the same attentional set, were presented successively in a rapid stream of distractors, if subjects increased the resources they devoted to T1 processing. This result is consistent with resource depletion accounts of the AB. However, Olivers, Spalek, Kawahara, and Di Lollo (2009) argue that Dux et al.'s results can be better explained by the relationship between $T 1$ and T2, and by target discriminability effects, rather than by the relationship between T1 and T3. Here, we find that manipulating the resources subjects devote to T1, either exogenously (target perceptual salience) or endogenously (target task relevance), affects T3 performance, even when T2 and target discriminability differences are controlled for. These results support Dux et al.'s conclusion that $T 1$ resource depletion underlies the $A B$.
\end{abstract}

The attentional blink (AB; Raymond, Shapiro, \& Arnell, 1992) refers to subjects' reduced ability to report the second of two targets (T2) in a rapid serial visual presentation (RSVP) stream of distractors, if this target appears within 200-600 msec of the first target (T1). Recently, the mechanisms responsible for this deficit have been under debate: We (Dux, Asplund, \& Marois, 2008) suggested that the $\mathrm{AB}$ reflects $\mathrm{T} 1$ resource depletion (RD); however, Olivers, Spalek, Kawahara, and Di Lollo (2009) state that we have "no basis for such a claim" (p. 214) and present alternative explanations for our results. Below, we present new data and arguments that refute Olivers et al.'s (2009) hypotheses and provide further support for RD accounts of the AB.

As we previously discussed (Dux et al., 2008), a classic theory of the $\mathrm{AB}$ postulates that the deficit arises because of limited-capacity resources being devoted to $\mathrm{T} 1$ processing at the expense of T2 (see, e.g., Chun \& Potter, 1995; Jolicœur \& Dell'Acqua, 1998). According to this theory, at short T1-T2 lags, T2 performance suffers because T1 is the subject of limited-capacity attentional processing when the second target appears, whereas at longer lags $\mathrm{T} 2$ performance is unimpaired because T1 processing is complete before the presentation of T2. Recently, such $\mathrm{RD}$ accounts have been challenged by the finding that in RSVP streams in which three targets, drawn from the same attentional set, are presented sequentially (uniform condition), performance for the third target does not differ from that for the first target. However, if the second target is replaced with a distractor from a different attentional set (varied condition), T3 performance is significantly impaired relative to that of T1 (see, e.g., Di Lollo, Kawahara, Ghorashi, \& Enns, 2005). According to the RD account, an $\mathrm{AB}$ (T1 performance $>\mathrm{T} 3$ performance) should be observed in both the uniform and varied conditions, because $\mathrm{T} 1$ will undergo the same limited-capacity processing in both conditions. The finding that an $\mathrm{AB}$ only occurs in the varied condition therefore appears inconsistent with $\mathrm{RD}$ theories.

To account for these three-target results, Di Lollo et al. (2005) and Olivers, van der Stigchel, and Hulleman (2007) proposed that it is not $\mathrm{T} 1$ processing, but rather processing of the distractor that directly follows $\mathrm{T} 1(\mathrm{~T} 1+1$ distractor), that gives rise to the $\mathrm{AB}$. The precise mechanisms hypothesized to be responsible for causing the $\mathrm{AB}$ in these input control (IC) accounts differ, with Di Lollo et al. suggesting that the $\mathrm{T} 1+1$ distractor disrupts an input filter, whereas Olivers et al. (2007) postulated that this stimulus instead triggers a suppressive response. However, both models suggest that the $\mathrm{AB}$ does not represent depletion of $\mathrm{T} 1$ resources, but rather imprecise attentional control upon presentation of the $\mathrm{T} 1+1$ distractor.

We (Dux et al., 2008) recently challenged these IC accounts by demonstrating that an $\mathrm{AB}$ can be observed not only in varied trials, but also under uniform conditions, if subjects increase the resources they devote to T1 processing. First, we replicated Di Lollo et al. (2005) by demonstrating that no $\mathrm{AB}$ was observed for $\mathrm{T} 3$ when three white letter targets were presented sequentially among a stream of white digit distractors (uniform condition), but that a T3 deficit was present when T2 was replaced with a white digit target (varied condition). To increase the attentional resources devoted to $\mathrm{T} 1$, we colored the target stimuli red, thus causing T1 to capture attention because of its abrupt color onset (Maki \& Mebane, 2006) relative to the preceding distractors. In comparison with the uniform condition of all white stimuli, this target color manipulation increased T1 accuracy and lowered T3 accuracy, thereby creating a blink, as predicted by the RD account of the AB. Finally, we confirmed that the T3 deficit was indeed an $\mathrm{AB}$, and not a working memory maintenance limitation, by manipulating the lag (the standard $\mathrm{AB}$ manipulation) between $\mathrm{T} 1$ and $\mathrm{T} 3$ under red-target uniform conditions, with T3 appearing either directly after T2 (short lag) or after

P. E. Dux, paul.dux@vanderbilt.edu 
five distractor items following T2 (long lag). We observed a much greater T3 deficit at the short relative to the long lag, indicating that the T1-T3 performance difference was most likely caused by an encoding bottleneck. From these results, we concluded that an $\mathrm{AB}$ can be observed under uniform conditions if sufficient resources are devoted to $\mathrm{T} 1$ processing. This finding fits remarkably well with $\mathrm{RD}$ theories but poses a serious challenge to IC accounts, because the latter theories predict that an $\mathrm{AB}$ should not be observed unless a $\mathrm{T} 1+1$ distractor impairs attentional control (Di Lollo et al., 2005; Olivers et al., 2007).

Olivers et al. (2009) agree with us that IC theories cannot account for ABs observed under uniform conditions, but they dispute whether the T3 deficits observed in our study (Dux et al., 2008) were indeed ABs. They have two main points of criticism: (1) performance levels on $\mathrm{T} 1$ and $\mathrm{T} 3$ are not directly related, but instead reflect separate relationships between T1 and T2 and between T2 and T3. Consequently, in their view, we have misinterpreted a correlation. (2) The enhanced T3 performance we observed at the long lag relative to the short lag reflects increased discriminability for $\mathrm{T} 3$ in the former condition rather than recovery from RD. Below, we present arguments and new data that refute the claims of Olivers et al. (2009). Furthermore, we not only replicate our previous finding that increasing $\mathrm{T} 1$ perceptual salience can trigger an $\mathrm{AB}$ under uniform conditions, we show that manipulations that affect $\mathrm{T} 1$ task relevance can also trigger an $\mathrm{AB}$. Taken together, these new findings provide strong support for $\mathrm{RD}$ accounts of the deficit.

\section{Do Subjects Trade Off T1 and T3 Performance?}

Olivers et al. (2009) suggest that differences in T1 and T3 performance between uniform and varied trials, in which all stimuli are the same color, do not reflect subjects trading off $\mathrm{T} 1$ and $\mathrm{T} 3$ performance, but are instead due to the influence of T2 on the two other targets. Put differently, Olivers et al. (2009) propose that T1 and T3 performance levels are not related and that the performance differences between uniform and varied conditions instead reflect $\mathrm{T} 2$ being drawn from a different category in the two conditions. In support of their hypothesis, Olivers et al.'s (2009) reanalysis of previous data (from Olivers et al., 2007) shows that $T 1$ performance was not affected when a third target was added to either two-target uniform or varied trials (as might be expected if targets compete for limited attentional resources), but that it was significantly decreased whenever a second target was inserted between $\mathrm{T} 1$ and $\mathrm{T} 3$. This finding provides evidence that $\mathrm{T} 2$ may indeed contribute to $\mathrm{T} 1$ performance differences between the uniform and varied conditions. However, this result is also somewhat consistent with RD accounts, since it could reflect a trade-off in performance between the first and second targets due to the temporal position of T2. More importantly, Olivers et al.'s (2009) criticism is not applicable to Dux et al.'s (2008) findings, because their conclusion that $\mathrm{T} 1$ and $\mathrm{T} 3$ performance levels are related is not based on the comparison of uniform and varied trials, but rather on the comparison of red- and white-target groups. Crucially, in both groups, T2 was drawn from the same category as the other targets and T3 was equally dis- criminable, because it was masked by stimuli of the same color. Thus, Dux et al.'s finding of enhanced T1 and reduced T3 performance in the red-target uniform condition relative to the white-target uniform condition (with a trend in the same condition in the varied trials) cannot be due to the influence of $\mathrm{T} 2$, because there were no differences between the conditions in the stimulus characteristics of $\mathrm{T} 2$, relative to its surrounding targets. Therefore, subjects do directly trade off $\mathrm{T} 1$ and $\mathrm{T} 3$ performance under threetarget RSVP conditions.

\section{EXPERIMENT 1 \\ Further Evidence of a T1-T3 Trade-Off}

Our previous study (Dux et al., 2008) provided evidence for a T1-T3 performance trade-off when the exogenous salience of T1 was manipulated. However, if RD accounts of the $\mathrm{AB}$ are correct, other $\mathrm{T} 1$ attentional manipulations should also affect T3 performance. Specifically, endogenous manipulations of target relevance should yield the same results. Moreover, if subjects trade off T1 and T3 performance under RSVP conditions, then, just as T1 performance increases and $\mathrm{T} 3$ performance decreases when $\mathrm{T} 1$ is attentionally emphasized, the reverse pattern of performance (lower for T1 and higher for T3) should be observed when T3 is prioritized.

To test this, we presented subjects with uniform and varied trials containing targets and distractors of the same color and manipulated the resources subjects devoted to $\mathrm{T} 1$ and $\mathrm{T} 3$ by varying each target's task relevance (see Figure 1A): One group of subjects had to report either all of the targets or just T1 (randomized within blocks; T1-relevant group), whereas the other group had to report either all of the targets or just T3 (T3-relevant group). Thus, for one group, T1 was relevant in $100 \%$ of the trials and T2 and T3 were relevant in only $50 \%$ of the trials, whereas for the second group, T3 was always relevant and $\mathrm{T} 1$ and T2 were relevant in only half of the trials. If resource depletion gives rise to the $\mathrm{AB}$ and subjects trade off performance between $\mathrm{T} 1$ and $\mathrm{T} 3$, performance should be reduced for T3 relative to T1 in T1-relevant trials. Conversely, T3-relevant trials should result in increased T3 performance and reduced T1 performance.

\section{Method}

Subjects. Forty-eight members (19 females, 29 males; mean age $=19$ years $)$ of the Vanderbilt University community participated. Subjects were allocated to either the T1-relevant or the T3relevant group (24 subjects each). All had normal or correctedto-normal vision. Three additional subjects were excluded for not following the task instructions.

Design. Two independent variables were manipulated: target relevance (T1- vs. T3-relevant group) and trial type (uniform vs. varied). Target relevance was manipulated between groups, whereas trial type was manipulated within groups across blocks of trials. T1, T2, and T3 accuracy levels were the measured variables.

Stimuli and Apparatus. The stimuli were white and appeared centrally on a gray background in Courier New font, subtending $1^{\circ}$ of visual angle at a viewing distance of approximately $57 \mathrm{~cm}$. In the uniform trials, the targets were three letters drawn from the alphabet, excluding I, L, O, Q, U, and V, and in the varied trials, they were two letters and a digit (2-9). The distractors were also selected from the 
A

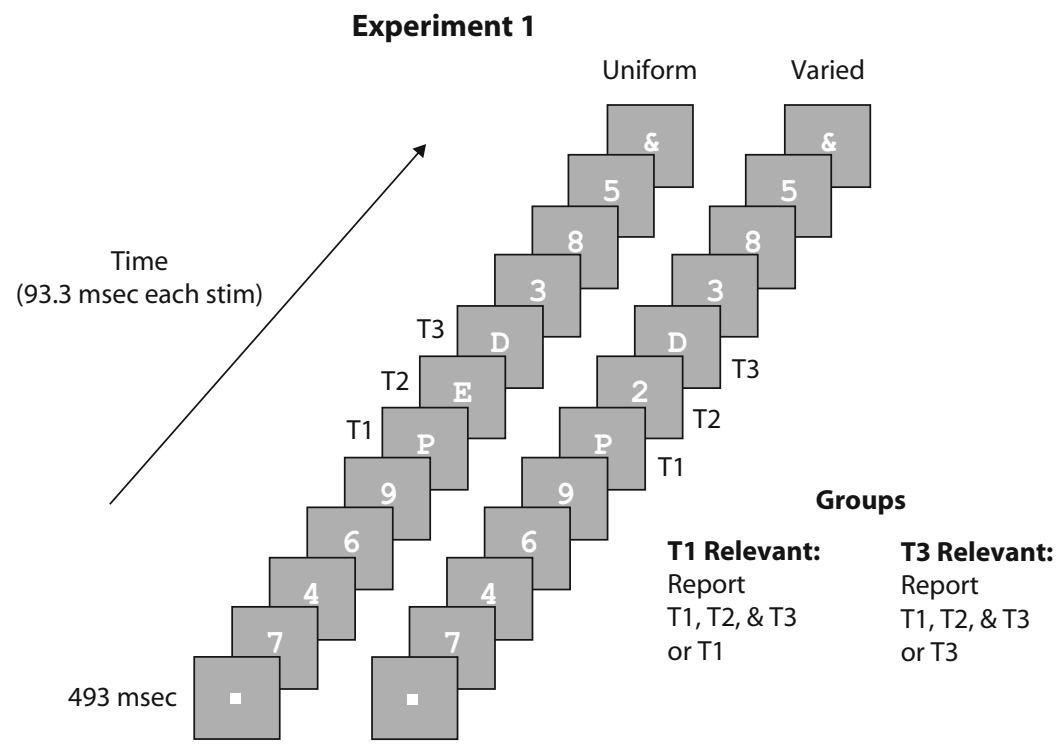

B

Experiment 2

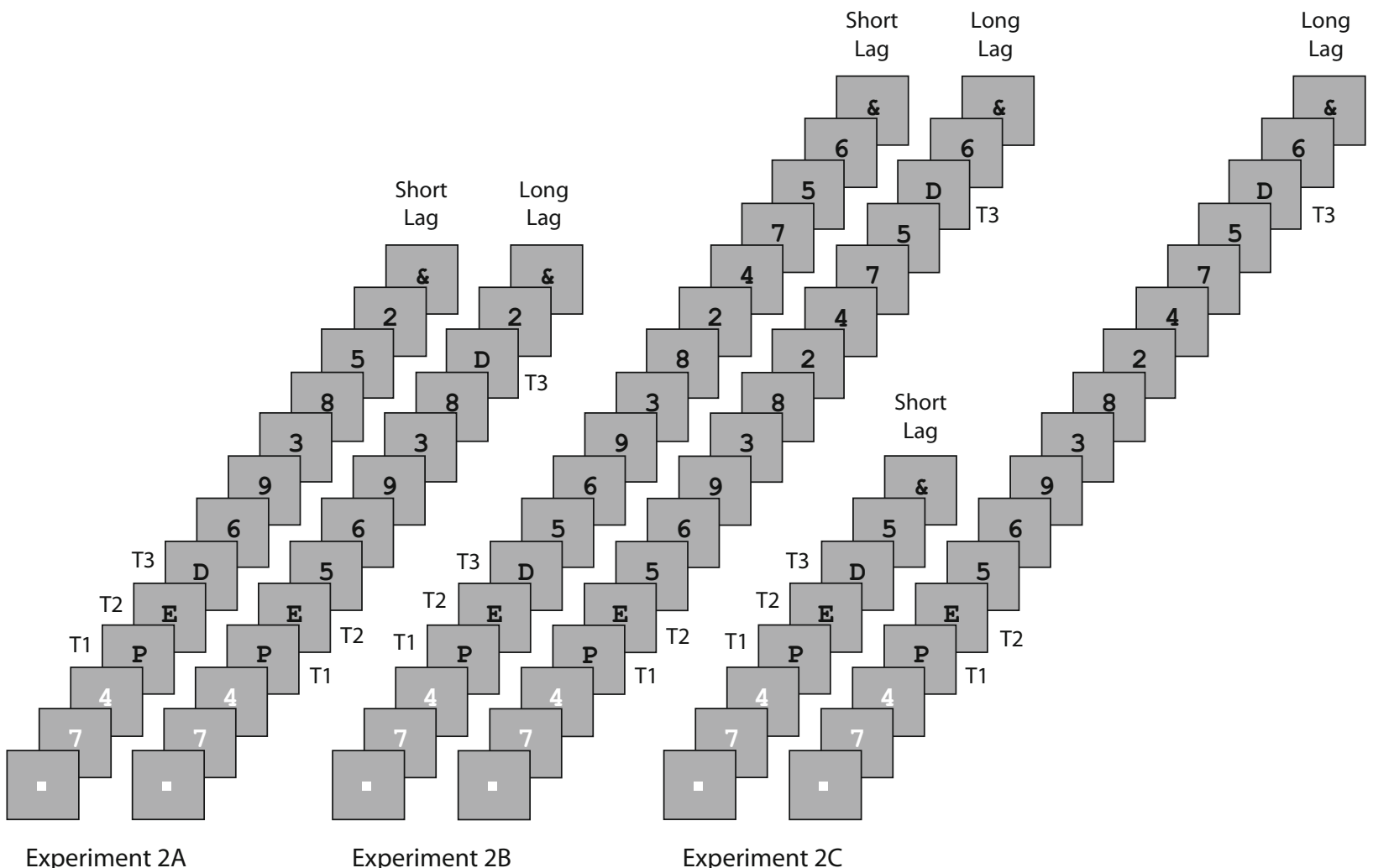

Figure 1. (A) Example RSVP streams for Experiment 1. (B) Example RSVP streams for Experiments 2A, 2B, and 2C. The black stimuli of the figure appeared in red in all of the experiments.

digits 2-9. No stimulus was repeated in the RSVP stream, and each trial included three targets, seven distractors, and an " \&" mask that appeared at the end of the stream. The stimuli were presented for $93.3 \mathrm{msec}$ each, with no interstimulus interval. T1 appeared equally often at Serial Positions 3-7 and was immediately followed by T2 and T3. The experiment was programmed using MATLAB and the Psychophysics Toolbox (Brainard, 1997; Pelli, 1997).
Procedure. The experiment was self-paced. Trials began with a white fixation square for $493 \mathrm{msec}$, followed by the RSVP stream. For all trials, subjects entered the identity of the target(s), without time pressure, via a keyboard at the end of each stream when visually prompted (e.g., "Target 1?"). To manipulate the resources subjects devoted to the targets, the task relevance of T1 and T3 was manipulated across groups of subjects. For the T1-relevant group, all trials 
involved reporting $\mathrm{T} 1$, whereas half of the trials also required report of T2 and T3. Similarly, for the T3-relevant group, all trials involved reporting T3, whereas only half required the report of all three targets. Importantly, subjects were only aware of whether they were to report one or three targets at the end of each trial. The presence of trials in which only one target required report was expected to lead subjects to emphasize the processing of either $\mathrm{T} 1$ or $\mathrm{T} 3$ over the other targets (T1 for the T1-relevant group and T3 for the T3-relevant group).

Each of the two test blocks contained 120 trials, the first 20 of which were practice and therefore not analyzed. Within groups, the trial type of the first block was counterbalanced across subjects.

\section{Results and Discussion}

Figure 2 displays mean target accuracy for the uniform and varied trials, plotted separately for the T1-relevant and T3-relevant groups (data from single-target trials were excluded from the analysis). The data were submitted to a 2 (target relevance) $\times 2$ (trial type) $\times 3$ (target number) mixed factorial ANOVA, which yielded a significant interaction between target relevance and target number $[F(2,92)=11.9, p<.001]$, indicating different patterns of performance, in both uniform and varied trials, across the three targets in the two groups.

Unsurprisingly, a T3 deficit (relative to T1) was found in the varied condition for the T1-relevant group $[t(23)=$ $10.6, p<.001]$. More importantly, a T3 deficit was also observed in the uniform condition for that group $[t(23)=$ $2.9, p<.008]$. Thus, by endogenously increasing the resources subjects devoted to $\mathrm{T} 1$ processing, we observed a $\mathrm{T} 3$ deficit under uniform conditions.

Strikingly, a reversed pattern of results was found in the T3-relevant group. Specifically, in the uniform condition, $\mathrm{T} 3$ accuracy was superior to that for $\mathrm{T} 1[t(23)=-2.9$,
T1 Relevant

Report T1, T2, \& T3

OR just T1

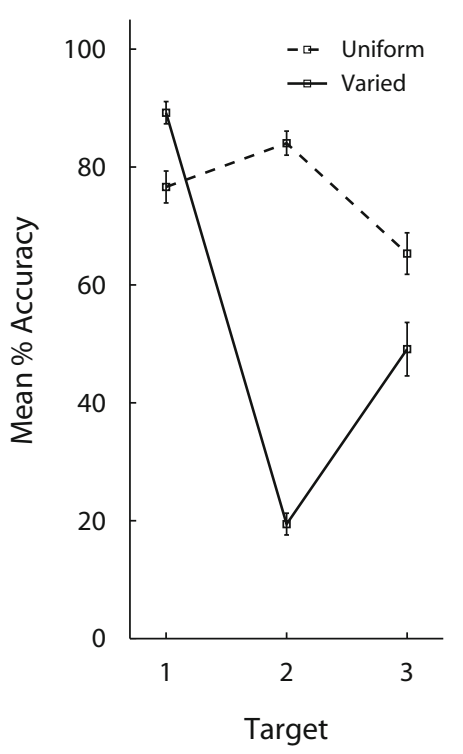

T3 Relevant

Report $\mathrm{T} 1, \mathrm{~T} 2, \& \mathrm{~T} 3$

OR just T3

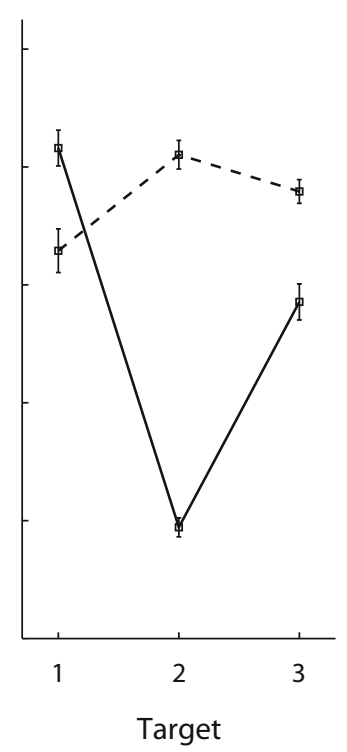

Figure 2. Mean T1, T2, and T3 accuracy in Experiment 1 as a function of trial type (uniform vs. varied), plotted separately for the T1-relevant and T3-relevant groups. $p<.009]$. Moreover, although a complete pattern reversal was not observed in the varied condition, the accuracy difference between $\mathrm{T} 1$ and $\mathrm{T} 3$ was nevertheless smaller here than that which was found in the varied condition for the T1-relevant group, because of a decrease in T1 accuracy and an increase in T3 performance $[t(46)=2.5$, $p<.02]$.

Taken together, these results show that subjects do trade off $\mathrm{T} 1$ and $\mathrm{T} 3$ performance under uniform conditions, since we found that simply varying the relevance of the targets-without manipulating their physical appearance - influenced the patterns of T1 and T3 accuracy. Importantly, these results were obtained without manipulating the $\mathrm{T} 2$ category, and there was no influence of target relevance on $\mathrm{T} 2(p \mathrm{~s}>.5)$. Thus, performance levels on T1 and T3 do appear to be directly related, contrary to Olivers et al.'s (2009) proposal.

\section{EXPERIMENT 2 Dux et al.'s (2008) Lag Effect Reflects RD}

To test whether the $\mathrm{T} 3$ deficit observed under uniform conditions was indeed an $\mathrm{AB}$ rather than the reflection of a working memory storage limitation (because of the requirement to maintain three targets), Dux et al. (2008) varied the temporal lag (the standard $\mathrm{AB}$ manipulation) between $\mathrm{T} 1$ and $\mathrm{T} 3$ under red-target uniform conditions. Their logic was that if depletion of T1 resources was responsible for the AB, T3 performance should recover with increasing lag, as more attentional resources were released from T1 processing. A T3 deficit was observed at both the short (targets presented successively) and long (T2 and T3 separated by five distractors) lags, but the deficit was much larger in the former condition. Thus, Dux et al. concluded that their uniform $\mathrm{T} 3$ deficit was an $\mathrm{AB}$, most likely caused by an encoding bottleneck.

Olivers et al. (2009) challenge this conclusion and instead argue that the lag effect we observed was simply due to $\mathrm{T} 3$ being more discriminable at the long than at the short lag, because only in the former condition was T3 an abrupt color onset. After replicating the lag effect from Dux et al. (2008) when the targets and distractors differed in color, Olivers et al. (2009) found no T3 performance difference between the short and long lags when the posttarget distractors were the same color as the targets. At first glance, Olivers et al.'s (2009) results suggest that our lag effect was indeed simply due to differences in T3 discriminability at the short and long lags. However, whereas Olivers et al. (2009) replicated the original result, they did not replicate our methodology: In addition to equating $\mathrm{T} 3$ discriminability, they randomly varied both lag and T1 stream position within blocks of trials, whereas we did not. This difference is important, because manipulations that increase the uncertainty of the temporal position of the targets in RSVP have been shown to increase AB magnitude (Chun \& Potter, 1995; Martens \& Johnson, 2005). Thus, Olivers et al.'s (2009) results could be due to equating T3 discriminability across the short and long lags or to the interaction of this manipulation and the other experimental characteristics they altered. 
To test whether discriminability alone was the important factor, we therefore equated T3 discriminability between the short- and long-lag trials using Dux et al.'s original design (Experiment 2A). In addition, we tested whether increasing the lag further reduced the T3 deficit, since a more complete recovery from the $\mathrm{AB}$ might be observed at very long lags (Experiment 2B). Finally, because subjects had to remember $\mathrm{T} 3$ for a longer period of time in the short-lag condition, we also tested whether having equal numbers of post-T3 distractors with the short and the long lags yielded the same results (Experiment 2C).

\section{Method}

Experiment 2 was identical to Dux et al.'s (2008) second experiment, except as specified below.

Subjects. Forty-two members ( 17 females, 25 males; mean age = 19 years) of the Vanderbilt University community participated. The subjects were excluded if they had low T1 accuracy (viz., $\leq 69 \%$; $n=5$ ), but this criterion did not change the pattern of results. Each experiment had 14 subjects.

Design. In all of the experiments, lag (short or long) was manipulated across blocks of trials. Short-lag trials contained three targets presented successively, whereas in long-lag trials five (Experiment $2 \mathrm{~A}$ ) or nine (Experiments $2 \mathrm{~B}$ and $2 \mathrm{C}$ ) distractors separated $\mathrm{T} 2$ and $\mathrm{T} 3$.

Stimuli and Apparatus. In both blocks of trials, three red letter targets appeared in an RSVP stream of digit distractors (see Figure 1B). Distractors prior to T1 were white, and those post-T1 were red; 8 distractors were present in Experiment 2A, 12 in Experiment 2B, 3 in the short-lag condition of Experiment 2C, and 12 in the long-lag condition of Experiment 2C. For short-lag trials, targets appeared at Serial Positions 3, 4, and 5. In the long-lag trials of Experiment 2A, targets appeared at Positions 3, 4, and 10, whereas in Experiments 2B and 2C they appeared at Positions 3, 4, and 14. Repeated presentations of distractors (necessitated by the length of the RSVP streams in Experiments 2B and 2C) occurred at least $650 \mathrm{msec}$ apart.
Procedure. All targets required report in each trial. One block of each trial type was completed (120 trials each, the first 20 of which were practice). Presentation order was counterbalanced across subjects.

\section{Results and Discussion}

Figure 3 shows mean target performance at the short and long lags, plotted separately for Experiments 2A, 2B, and $2 \mathrm{C}$. The data from the experiments were submitted to separate 2 (lag) $\times 3$ (target number) repeated measures ANOVAs, each of which yielded a significant two-way interaction $(F \mathrm{~s}>4.9, p \mathrm{~s}<.02)$, demonstrating that in all of the experiments, performance across the three targets was different in the two lag conditions.

In Experiment 2A, significant $\mathrm{T} 3$ deficits were present at both the short lag $[t(13)=4.7, p<.001]$ and the long lag $[t(13)=2.7, p<.02]$, but this T1-T3 difference was much smaller in the latter condition $[t(13)=2.2, p<$ $.05]$. Experiment 2B demonstrated that the T3 deficit in the long-lag condition was abolished when the T1-T3 lag was increased (from lag 7 to 11). Here, whereas there was still a significant T3 deficit at the short lag $[t(13)=4.8$, $p<.001]$, this deficit was absent at the long lag $(p=.18)$. Finally, Experiment 2C confirmed that T3 performance differences between the short and long lags were not due to a larger number of distractors following $\mathrm{T} 3$ in the former condition, because when a single distractor followed T3 at both lags, the results mirrored those from Experiment 2B: a T3 deficit at the short $[t(13)=2.7, p<.001]$, but not the long ( $p=.34)$, lag.

Taken together, these results replicate those from Dux et al.'s (2008) second experiment and indicate that a lag effect can be obtained when controlling for T3 discriminability differences between the lags. Evidently, Olivers
Experiment 2A

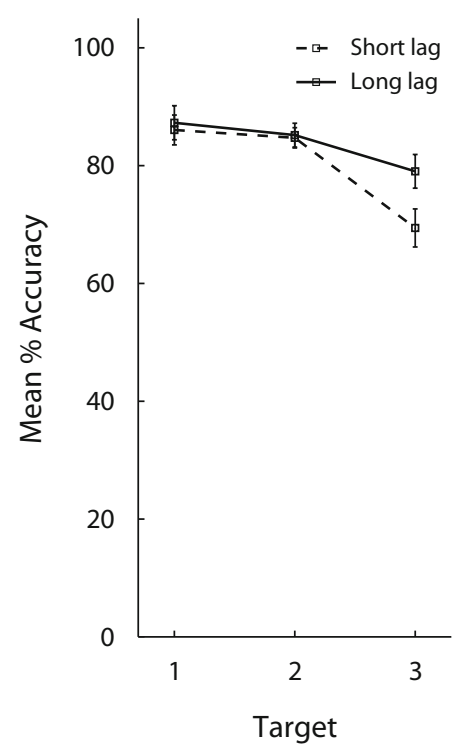

Experiment 2B

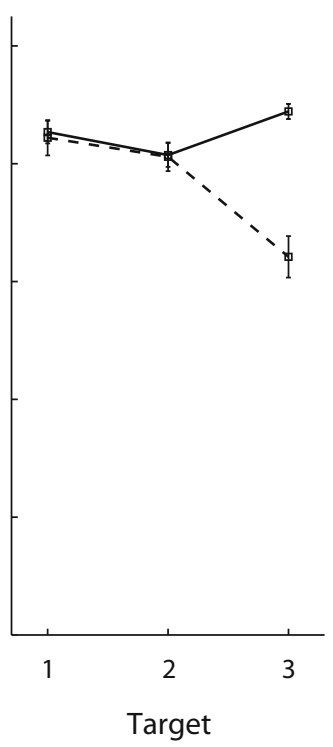

Experiment 2C

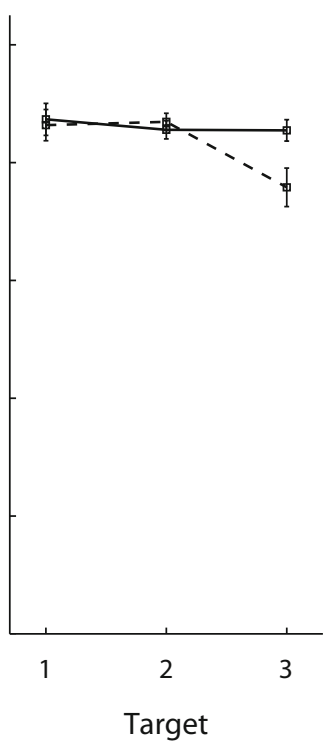

Figure 3. Mean T1, T2, and T3 accuracy as a function of T1-T3 lag (short vs. long), plotted separately for Experiments $2 \mathrm{~A}, 2 \mathrm{~B}$, and $2 \mathrm{C}$. 
et al.'s (2009) failure to find an attenuated T3 deficit at the long lag reflects the interaction of their color manipulation with the other methodological differences that they employed relative to Dux et al.'s experiment. These results also confirm that Dux et al.'s uniform T3 deficit is not due to a working memory maintenance limitation, because $\mathrm{T} 3$ performance was worse at the short than at the long lag, even though both conditions contained the same number of targets.

\section{CONCLUSIONS}

Olivers et al. (2009) claim that Dux et al. (2008) provided no evidence supporting the hypothesis that the depletion of attentional resources by $\mathrm{T} 1$ gives rise to the $\mathrm{AB}$. They suggest that subjects do not trade off performance between T1 and T3 under uniform conditions, and that Dux et al.'s lag effect in red-target uniform trials was the result of differences in T3 discriminability at the short and long lags. In short, they postulate that the uniform T3 deficit found by Dux et al. was not an AB.

Here, we have not only shown that our uniform T3 deficit is indeed an $\mathrm{AB}$, but also that it can be produced regardless of whether the attentional resources devoted to T1 are manipulated exogenously or endogenously. In Experiment 1, we demonstrated that three-target RSVP performance could be strongly affected by manipulating the task relevance of the targets. When $\mathrm{T} 1$ required report on $100 \%$ of the trials and $\mathrm{T} 2$ and $\mathrm{T} 3$ required report on only $50 \%$ of the trials, T1 accuracy increased and T3 accuracy decreased. When T3 accuracy was emphasized, the opposite pattern of results was observed. In addition, Experiment 2 demonstrated that the larger T3 deficit observed in uniform trials at the short lag than at the long lag was not due to T3 discriminability differences between these conditions. This result also demonstrates that a working memory maintenance limitation cannot explain the $\mathrm{AB}$ observed in the uniform trials. Taken together, these findings reinforce those of Dux et al. (2008) and demonstrate that subjects do trade off target performance in uniform trials. Our results therefore strongly support RD accounts of the $\mathrm{AB}$, because they demonstrate that attentional prioritization of $\mathrm{T} 1$ impairs $\mathrm{T} 3$ processing.

It is worth noting, however, that the flexibility of these attentional resources may not be unlimited, since the results from the varied condition of Experiment 1 demonstrate that, although emphasizing T3 influences both T1 and T3 performance, it does not always lead to performance for the third target being superior to that for T1 (as was the case in the T3-relevant uniform trials). In addition, our results do not rule out the possibility that selection limitations may contribute to the AB deficit. However, our findings (Dux et al., 2008; the present article) do place severe constraints on IC accounts, because they show that a T1 processing bottleneck plays a vital role in the generation of the AB. Evidently, the T1 RD hypothesis is well and truly back in the saddle.

\section{AUTHOR NOTE}

NIMH Grant R01 MH70776 to R.M. supported this work. Correspondence related to this article may be sent to P. E. Dux, Department of Psychology, Vanderbilt University, 428 Wilson Hall, 111 21st Avenue South, Nashville, TN 37203 (e-mail: paul.dux@vanderbilt.edu).

\section{REFERENCES}

Brainard, D. H. (1997). The Psychophysics Toolbox. Spatial Vision, 10, 433-436.

Chun, M. M., \& Potter, M. C. (1995). A two-stage model for multiple target detection in rapid serial visual presentation. Journal of Experimental Psychology: Human Perception \& Performance, 21, 109-127.

Di Lollo, V., Kawahara, J.-I., Ghorashi, S. M. S., \& Enns, J. T. (2005). The attentional blink: Resource depletion or temporary loss of control? Psychological Research, 69, 191-200.

Dux, P. E., Asplund, C. L., \& Marois, R. (2008). An attentional blink for sequentially presented targets: Evidence in favor of resource depletion accounts. Psychonomic Bulletin \& Review, 15, 809-813.

Joliceleur, P., \& Dell'ACQua, R. (1998). The demonstration of shortterm consolidation. Cognitive Psychology, 36, 138-202.

MaKi, W. S., \& Mebane, M. W. (2006). Attentional capture triggers an attentional blink. Psychonomic Bulletin \& Review, 13, 125-131.

Martens, S., \& Johnson, A. (2005). Timing attention: Cuing target onset interval attenuates the attentional blink. Memory \& Cognition, 33, 234-240.

Olivers, C. N. L., Spalek, T. M., Kawahara, J.-I., \& Di Lollo, V. (2009). The attentional blink: Increasing target salience provides no evidence for resource depletion. A commentary on Dux, Asplund, and Marois (2008). Psychonomic Bulletin \& Review, 16, 214-218.

Olivers, C. N. L., van der Stigchel, S., \& Hulleman, J. (2007). Spreading the sparing: Against a limited-capacity account of the attentional blink. Psychological Research, 71, 126-139.

Pelli, D. G. (1997). The VideoToolbox software for visual psychophysics: Transforming numbers into movies. Spatial Vision, 10, 437-442.

Raymond, J. E., Shapiro, K. L., \& Arnell, K. M. (1992). Temporary suppression of visual processing in an RSVP task: An attentional blink? Journal of Experimental Psychology: Human Perception \& Performance, 18, 849-860.

(Manuscript received July 30, 2008; revision accepted for publication August 19, 2008.) 